\title{
Desempeño de una Oficina de Transferencia de Tecnología en el contexto de gestión de patentes: Estudio de caso de la OTT de la Benemérita Universidad Autónoma de Puebla \\ Performance of a Technology Transfer Office in the context of patent management: Case study of the OTT of the Benemerita Autonomous University of Puebla
}

Antonio Solís Lima ${ }^{1,2}$, Catalina Ovando ${ }^{1}$, Emmanuel Olivera Pérez ${ }^{1}$ y Miguel Ángel Rodríguez Lozada $^{2}$

Palabras clave: licenciamiento; OTT; patentes; spinoffs; transferencia de tecnología; BUAP; propiedad intelectual; propiedad industrial Keywords: licensing; TTO; patents; spinoffs; technology transfer; BUAP; intellectual property; industrial property

Recibido en: 30-09-2019 / Aceptado en: 05-12-2019

\section{Resumen}

Introducción: Con la finalidad de detonar la transferencia de conocimiento, el gobierno mexicano, ha establecido la política pública de la creación de Oficinas de Transferencia de Tecnología (OTT); sin embargo, esta política ha mostrado ser ineficaz debido a la inconsistencia en la permanencia de la mayoría de las OTT mexicanas. A pesar de esto, existen algunas OTT que han sobresalido; tal es el caso de la OTT de la Benemérita Universidad Autónoma de Puebla (OTT-BUAP) que fue establecida en el 2011. Dicha oficina ha posicionado a esta universidad en los primeros lugares a nivel nacional en el contexto de generación de patentes, razón para que esta OTT haya sido el objeto de análisis de este estudio para dar respuesta a la siguiente pregunta de esta investigación: ¿Cómo ha logrado la OTT-BUAP posicionarse dentro de las mejores OTT en México, en el ámbito de gestión de patentes, durante el periodo 2011-2018?

El objetivo general de esta investigación es contribuir al conocimiento del especialista en transferencia y comercialización de tecnología que se dedica a la gestión de las OTT Universitarias. En cuanto a la revisión de la literatura, se encontró que este tema relacionado con el desempeño de las OTT mexicanas en el ámbito de gestión de patentes ha sido poco estudiado, por lo cual se justifica esta investigación.

Método: Esta investigación se considera de tipo exploratorio, cualitativo, descriptivo y transversal, la cual consistió en aplicar la metodología del estudio del caso a la OTT-BUAP, basándose en seis proposiciones formuladas a partir de fuentes secundarias.

\footnotetext{
${ }^{1}$ Universidad Popular Autónoma del Estado de Puebla

${ }^{2}$ Tecnológico Nacional de México/Instituto Tecnológico de Apizaco, México. Email: antonio.sl@apizaco.tecnm.mx

(C) Universidad De La Salle Bajío (México)
} 
Resultados: Se halló que de los procesos de protección de propiedad intelectual, consultoría, licenciamiento y la creación de spinoffs, solo el proceso de protección de propiedad intelectual es el que se encuentra en etapa de maduración en dicha OTT. También, se halló que la OTT-BUAP cuenta con un equipo experto en la redacción de patentes, el cual fue contratado específicamente, para la creación e impulso de dicha OTT.

Discusión o Conclusión: Según los resultados encontrados en esta investigación, la razón por la que la OTT-BUAP se ha posicionado dentro de las mejores OTT en México en el periodo 20112018, se debe, en gran medida, a que cuenta con un proceso de patentamiento eficaz, basado en una normativa equitativa en la repartición de regalías apoyado en un plan estratégico de incentivos para motivar a los investigadores a proteger los resultados de sus investigaciones. Dicho proceso fue diseñado y gestionado por un investigador proveniente del Instituto Mexicano de la Propiedad Industrial, experto en protección de la propiedad intelectual, contratado por la BUAP, específicamente, para conformar un equipo de trabajo profesional para la redacción de patentes y otras tareas relacionadas con la transferencia y comercialización de la tecnología proveniente de la BUAP.

\begin{abstract}
Introduction: In order to detonate the transfer of knowledge, the Mexican government has established the public policy of the creation of Technology Transfer Offices (OTT); however, this policy has proved ineffective due to the inconsistency in the permanence of most Mexican TTOs. Despite this, there are some OTTs that have excelled; such is the case of the OTT of the Benemerita Autonomous University of Puebla (OTT-BUAP) that was established in 2011. This office has positioned this university in the first places nationwide in the context of patent generation, reason for that this OTT has been the object of analysis of this study to answer the following question of this research: How has the OTT-BUAP managed to position itself within the best OTT in Mexico, in the field of patent management, during the 2011-2018 period?

The general objective of this research is to contribute to the knowledge of the specialist in technology transfer and commercialization that is dedicated to the management of University OTTs.
\end{abstract}


Regarding the review of the literature, it was found that this issue related to the performance of Mexican TTOs in the field of patent management has been poorly studied, which is why this research is justified.

Method: This research is considered exploratory, qualitative, descriptive and transversal, which consisted in applying the case study methodology to the OTT-BUAP, based on six propositions made from secondary sources.

Results: It was found that of the processes of intellectual property protection, consulting, licensing and the creation of spinoffs, only the process of intellectual property protection is the one that is in the maturation stage in said OTT. It was also found that the OTT-BUAP has an expert team in the drafting of patents, which was hired, specifically, for the creation and promotion of said OTT.

Discussion or Conclusion: According to the results found in this investigation, the reason why the OTT-BUAP has positioned itself among the best OTTs in Mexico in the 2011-2018 period, is largely due to the fact that it has an effective patenting process, based on equitable regulations on the distribution of royalties supported by a strategic incentive plan to motivate researchers to protect the results of their research. This process was designed and managed by a researcher from the Mexican Institute of Industrial Property, an expert in intellectual property protection, hired by BUAP, specifically, to form a professional teamwork for the drafting of patents and other tasks related to the transfer and commercialization of technology that comes from the BUAP.

\section{Introducción}

Hoy en día, existe una gran cantidad de publicaciones sobre gestión, organización, función y rendimiento de las OTT. Aunado a esto, han proliferado diferentes acrónimos para identificar este tipo de oficinas, ejemplos de ello, tenemos a Pedraza y Velázquez (2013), quienes las reconocen como Unidades de Vinculación y Transferencia de Conocimiento (UVTC); Pérez (2016), las identifica como Oficinas de Transferencia de Conocimiento (OTC); Cruz Novoa (2016), las describe como Oficinas de Transferencia y Licenciamiento (OTL); Pérez y Calderón (2014) las reconocen como Oficinas de Transferencia de Tecnología (OTT); y Alvarado (2018), las describe como Oficinas de Transferencia de Tecnología Universitarias (OTTUS). En este documento se 
usará el acrónimo OTT puesto que es el que más se usa en el contexto mundial, y además abarca las OTT de los Centros de Investigación (CI).

Estas OTT establecidas en universidades, CIs y otras organizaciones públicas y privadas dedicadas a la investigación, desempeñan un papel importante para llevar los resultados de los investigadores al mercado mediante mecanismos de transferencia, tales como, acuerdos de colaboración, licenciamientos, consultorías, o mediante la creación de empresas spinoffs (Granieri \& Basso, 2019).

Por lo que respecta a la creación de las OTT, diferentes estudios muestran que la creación de estas en el mundo, es un proceso que data desde el siglo pasado. A este respecto, Estrada (2009) señala que la primera OTT fue establecida en EE. UU. por el Instituto Tecnológico de Massachusetts en 1932; no obstante, el impulso masivo para la creación de las OTT Universitarias en ese país se dio a partir de 1980, después del establecimiento de la ley de BayhDole (Estrada, 2009). Así mismo, Arundeep (2016) menciona que dicha ley fue establecida en respuesta a la amenaza económica que tenía EE. UU., principalmente, de Japón, y también debido a los miles de patentes que poseía, en ese entonces, el gobierno americano que no estaban siendo comercializadas. El objetivo de dicha ley fue detonar la economía americana a través de la comercialización de las patentes provenientes de la investigación (Arundeep, 2016). Además, Gotkin (2012) menciona que esta ley otorgó a las universidades de EE. UU. la titularidad de las patentes resultantes de investigaciones financiadas por el gobierno federal a cambio de que las universidades trataran de comercializarlas.

Este evento en EE. UU. motivó a que otros países empezaran a impulsar la creación de OTT en sus universidades. En el contexto latinoamericano, tomando como referencia a Brasil, Chile y México por tener los sistemas de innovación más avanzados en la región (Solleiro \& Escalante, 2009); el impacto fue el siguiente.

Por lo que se refiere a Brasil, Dos Santos \& Torkomian (2013) mencionan que las universidades y los centros de investigación empezaron a crear sus OTT a partir de 1990, sin embargo, el auge se dio a partir del 2006, incrementadose de la siguiente manera: en el 2006 se tenían 43 OTT; para el 2007 se incrementó a 72 OTT; 2008, 101 OTT; 2009, 156 OTT; 2010, 164 OTT y para el 2011, Brasil ya tenía 176 OTT. Además, esto generó un incremento de las solicitudes y obtención de patentes de los brasileños tanto en Brasil como en el extranjero (Dos Santos \& Torkomian, 2013). 
De igual modo, en Chile, según con el informe de la Corporación de Fomento a la Producción (CORFO) publicado en el 2016, se menciona que en ese país en la década de los 90s, algunas universidades chilenas ya habían empezado a proteger los resultados de sus investigaciones, pero fue hasta el 2012 cuando la creación de las Ofícinas de Transferencia y Licenciamiento (OTL) se empezarón a formalizar. En dicho informe se señala que en el 2016, Chile ya contaba con 29 OTL establecidas por diferentes universidades, las cuales ya habían impactado la producción de protección de propiedad intelectual en ese país (CORFO, 2016).

En el caso de México, según Rojas (2017), la creación de las OTT surge con la finalidad de incrementar el número de empresas y entes públicos que demandan la generación de ideas y soluciones innovadoras para su comercialización. Y de esta forma, dar cumplimiento al compromiso del gobierno federal mexicano de fortalecer la innovación empresarial, lo cual esta estipulado en el Programa Nacional de Innovación del 2011 (CII, 2011).

En este mismo contexto, Medellín (2015) señala que la Secretaria de Economía (SE) y el Consejo Nacional de Ciencia y Tecnología (CONACyT), a través del "Fondo Sectorial de Innovación Secretaría de Economía-CONACyT (FINNOVA)" establecieron el programa para impulsar la creación de OTT en México a partir del 2011.

Es importante hacer mención, que este programa de creación y certificación de OTT ha sido gestionado de forma irregular a desde su creación, ya que dicho programa se mantuvo vigente en el periodo 2011-2014, posteriormente fue suspendido durante el periodo 2015-2016; y finalmente, el gobierno mexicano lo volvió habilitar en el 2017 (SE-CONACyT, 2017; SECONACyT, 2018). Esta inconsistencia de la funcionalidad de dicho programa, ha traído como consecuencia una disminución en el número de OTT reconocidas por el CONACyT. En el 2014, el número de OTT reconocidas por el CONACyT era de 117; sin embargo, en el informe de dicho organismo en el 2018, se reportan solamente 75 OTT (OTT-CONACyT, 2018).

En la Tabla 1 se muestra el resultado de la distribución de la certificación por el tipo de organización de las OTT ante el CONACyT, donde se puede apreciar que las OTT universitarias disminuyeron su participación en el 2018. Así mismo, en la Tabla 2 se muestran las 18 universidades que han mantenido la certificación de sus OTT ante el CONACyT en el periodo 2011-2018. Las cuales son pocas comparadas con la cantidad potencial de universidades que podrían tener su OTT en México. Según Mendoza (2018), en México, hasta el 2017 existían un promedio de 3,145 IES. 
A pesar de las vicisitudes que ha presentado esta política pública en México sobre el establecimiento de OTT durante el periodo 2011-2019, ha habido algunas OTT que se han mantenido activas y productivas. Entre ellas, se encuentra la OTT-BUAP, la cual se posicionó en el tercer lugar a nivel nacional en el contexto de generación de propiedad industrial en el 2018 (Heraldo, 2018), razón importante para que dicha OTT sea el objeto de estudio de esta investigación para encontrar las causales de su éxito y tomarlas como referencia para el establecimiento y operación de otras OTT en México.

Por otra parte, en cuanto a la revisión de la literatura de esta investigación, se encontró que este tema relacionado con el desempeño de las OTT mexicanas en el ámbito de gestión de patentes ha sido poco estudiado, por lo cual se justifica esta investigación.

Tabla 1. Distribución de las OTT certificadas por tipo de organización en el periodo 20112018.

Table 1. Distribution of certified TTO by type of organization in the period 2011-2018.

\begin{tabular}{|lcc|}
\hline Tipo de OTT & $\mathbf{2 0 1 4}$ & $\mathbf{2 0 1 8}$ \\
\hline Centros de investigación & 6 & 1 \\
Centros públicos de investigación & 14 & 17 \\
Gobierno & 6 & 3 \\
Instituciones de Educación Superior Pública & 23 & 18 \\
Instituciones de Educación Superior Privadas & 14 & 6 \\
Empresas o consultorías privas & 54 & 30 \\
\hline Total & $\mathbf{1 1 7}$ & $\mathbf{7 5}$ \\
\hline
\end{tabular}

Fuente: Elaboración propia con base en resultados FINNOVA (2014), OTT-CONACYT (2018). Source: Own elaboration based on results FINNOVA (2014), OTT-CONACYT (2018).

El objetivo general de esta investigación es contribuir al conocimiento del especialista en transferencia y comercialización de tecnología que se dedica a la gestión de Oficinas de Transferencia de Tecnología Universitarias.

El objetivo específico de esta investigación es encontrar como ha logrado la OTT-BUAP posicionarse dentro de las mejores OTT en México, en el ámbito de gestión de patentes, durante el periodo 2011-2018. 
Tabla 2. Universidades que han mantenido la certificación de sus OTT ante el CONACyT en el periodo 2011-2018.

Table 2. Universities that have retained their OTT certification by CONACyT in the period 2011-2018.

\begin{tabular}{|cc|}
\hline $\mathbf{1}$ & Benemérita Universidad Autónoma de Puebla \\
$\mathbf{2}$ & Instituto Politécnico Nacional (Unidad Politécnica para el Desarrollo y la Competitividad Empresarial) \\
$\mathbf{3}$ & Instituto Tecnológico y de Estudios Superiores de Monterrey \\
$\mathbf{5}$ & Instituto Tecnológico y de Estudios Superiores de Monterrey (REGIONAL CENTRO) \\
$\mathbf{6}$ & Instituto Tecnológico y de Estudios Superiores de Occidente, A.C. \\
$\mathbf{7}$ & Operadora de Bienes y Servicios de la Universidad Politécnica de Pachuca, S.C. \\
$\mathbf{8}$ & Promoción de la Cultura y la Educación Superior del Bajío A.C. (Universidad Iberoamericana León) \\
$\mathbf{9}$ & Tecnológico Nacional de México-Centro Nacional de Investigación y Desarrollo Tecnológico \\
$\mathbf{1 0}$ & Universidad Autónoma de Ciudad Juárez \\
$\mathbf{1 1}$ & Universidad Autónoma de Sinaloa \\
$\mathbf{1 2}$ & Universidad Autónoma de Tamaulipas \\
$\mathbf{1 3}$ & Universidad Autónoma del Estado de Morelos \\
$\mathbf{1 4}$ & Universidad Autónoma Metropolitana, Unidad Cuajimalpa \\
$\mathbf{1 5}$ & Universidad de Sonora \\
$\mathbf{1 6}$ & Universidad del Mayab, S.C. \\
$\mathbf{1 7}$ & Universidad Estatal de Sonora \\
$\mathbf{1 8}$ & Universidad Nacional Autónoma de México \\
\hline
\end{tabular}

Fuente: Elaboración propia con base en resultados FINNOVA (2014), OTT-CONACYT (2018).

*La certificación de cada OTT ante el CONACyT se debe de refrendar cada dos años después de su creación.

Source: Own elaboration based on results FINNOVA (2014), OTT-CONACYT (2018).

*The certification of each OTT by CONACyT must be endorsed every two years after its creation.

Este trabajo de investigación se considera de corte cualitativo, exploratorio, descriptivo y transversal, el cual consistió en aplicar la metodología del caso de estudio a la OTT-BUAP mediante entrevistas e investigación en documentos; actividades realizadas por el investigador principal de este trabajo durante una estancia en dicha OTT.

Es importante hacer mención que no toda la investigación que se efectúa al interior de las universidades debe ser protegida para su comercialización, sino que también, tal como lo hacen notar Pagano y Rossi (2017), existe investigación en las diferentes áreas de conocimiento que se debe continuar promoviendo y divulgando de manera abierta para seguir construyendo el conocimiento.

\section{$\underline{\text { Revisión de la literatura }}$}

Las OTT tienen razón de ser desde el momento que se les delegó la tarea de participar en el desarrollo económico de su entorno, a través de la transferencia del conocimiento o tecnología hacia otras organizaciones (Alvarado, 2018). 
Ahora bien, para facilitar la tarea del establecimiento de las OTT, diferentes equipos de trabajo de organismos nacionales e internacionales han publicado varios documentos y manuales para ser tomados como referencia.

En el 2011, Mandrieux a través de la Organización Mundial de la Propiedad Industrial (OMPI) emitió el documento "Guía práctica para la creación y la gestión de Oficinas de Transferencia de Tecnología en universidades y centros de investigación”, con el propósito de ofrecer a las entidades antes mencionadas, un instrumento de referencia para el establecimiento de una OTT (Manderieux, 2011).

De manera similar, en el 2011 un equipo de trabajo de la fundación Implementación, Diseño, Evaluación y Análisis de Políticas Públicas (IDEA) con apoyo del gobierno Británico e Irlanda del Norte, entre otros, desarrollaron el documento "La transferencia de conocimiento. Mejores prácticas internacionales para el diseño de un programa de transferencia de conocimiento en México", con el objeto de ser utilizado por la SE y el CONACyT en la creación e implementación del Programa Nacional para la Transferencia de Conocimiento (IDEA, 2011).

De igual modo, en el 2012, un equipo de trabajo de la SE y el CONACyT emitieron el documento "Guía para la certificación de una Oficina de Transferencia de Conocimiento (OT)", con la finalidad de acreditar aquellas OTT mexicanas que cumplieran con los requisitos necesarios para que pudieran funcionar como agentes de Transferencia de Tecnología (TT) (SECONACyT, 2012).

Ahora bien, debido a que esta investigación se considera del tipo exploratorio, se tomaron como referencia los tres documentos antes mencionados para formular las seis proposiciones que sirvieron de base para la realización de esta investigación, las cuales se describen en el apartado de metodología en este documento. Además, dichas proposiciones permitieron realizar contrastes de los hallazgos encontrados en la investigación.

\section{Metodología}

La metodología aplicada para llevar a cabo esta investigación fue la del estudio de caso, la cual nos sirve para interpretar fenómenos sociales, y que ha sido aplicada, entre otros, por Villegas y Cárdenas (2018) para el estudio de caso de la Universidad EAFIT. Este método del caso de estudio propuesto por Yin (1994), quien lo define como: "un cuestionamiento empírico que investiga un fenómeno contemporáneo a profundidad en un contexto de la vida real, 
especialmente cuando las fronteras entre el fenómeno y el contexto no son claramente evidentes," nos permitió encontrar características y rasgos importantes de la OTT-BUAP.

El tipo de diseño para el caso de estudio, tomando en cuenta la clasificación de Yin (2009), fue individual y holístico, ya que se analizó exclusivamente la OTT-BUAP en un solo contexto para el periodo 2011-2018.

\section{Información general de la OTT-BUAP en México}

La OTT-BUAP fue creada en el año 2011 por la parte directiva de dicha universidad, y reconocida por el CONACyT y la SE en el año 2012.

Actualmente, dicha OTT se desenvuelve en un ambiente universitario que de acuerdo con el primer informe de gobierno del rector de la BUAP, Alfonso Esparza, esta universidad comprende una matrícula promedio de 77,223 estudiantes, atendidos por un promedio de 3,928 profesores, de los cuales 664 son miembros del Sistema Nacional de Investigadores (SNI), además dicha Universidad cuenta con 214 cuerpos académicos (BUAP, 2018).

En relación con la protección de la propiedad intelectual, se encontró que en el periodo 2011-2019, se han protegido 29 obras ante el Instituto Nacional del Derecho de Autor vía la OTT_BUAP (OCT-INDAUTOR, 2019). Así mismo, en el contexto de protección industrial, se identificarón 234 solicitudes de patente ingresadas al IMPI vía la OTT-BUAP en el periodo 2011-2018 (OCT-PAT, 2019).

Por otro lado, de acuerdo con cifras dadas a conocer por el Instituto Mexicano de la Propiedad Industrial (IMPI), dentro de las diez Instituciones de Educación Superior (IES) mexicanas que más solicitudes de patente presentaron durante el periodo 2013-2018 fueron: la Universidad Nacional Autónoma de México con 299, el Instituto Politécnico Nacional con 248 y la BUAP con 169 (IMPI, 2018).

Los servicios que actualmente ofrece la OTT-BUAP a su comunidad universitaria, entre otros, son la gestión de patentes, marcas, diseños industriales, modelos de utilidad, derechos de autor, derechos de obtentor; estudios de vigilancia tecnológica; libertad de operación de patentes, y análisis de viabilidad tecnológica de proyectos. 


\section{Método para el estudio de caso}

A este respecto, Villegas y Cárdenas (2018) mencionan que los estudios de caso constan de cinco pasos: 1. diseño del estudio de caso, 2. preparación de la recolección de la información, 3. la recolección de la información, 4. el análisis de la información, 5. la realización del informe. Estos cinco pasos fueron considerados para esta investigación.

\section{Primer paso - Diseño del estudio de caso}

De acuerdo a la metodología de Yin (2009, p. 27), para los diseños de estudios de caso existen cinco componentes importantes que son: las preguntas de estudio; sus proposiciones, si las hay; sus unidades de análisis; la lógica que enlaza los datos con las proposiciones; y los criterios para interpretar los hallazgos. Estos elementos fueron considerados y desarrollados de la siguiente manera.

\section{Pregunta de estudio}

Con el estudio de caso de la OTT-BUAP se pretende dar respuesta a la pregunta de investigación: ¿Cómo ha logrado la OTT-BUAP posicionarse dentro de las mejores OTT en México, en el ámbito de gestión de patentes durante el periodo 2011-2018?

\section{Proposiciones}

Derivado de la revisión de literatura de esta investigación, se formularon las siguientes seis proposiciones que motivaron el desarrollo del caso.

$\mathbf{P}_{\mathbf{1}}$ : La estructura organizacional de una OTT es un factor importante que influye en la funcionalidad de la misma. A este respecto, la Organización para la Cooperación y el Desarrollo Económicos (OCDE), reconoce tres modelos de establecimiento para las OTT: oficinas dedicadas (internas, externas), oficinas administrativas, y oficinas proveedoras externas (públicas o privadas); de los cuales, la OCDE identifica que las OTT del tipo dedicada-interna son las que predominan, al menos, en aquellos países que conforman la OCDE (IDEA, 2011; OECD, 2003).

$\mathbf{P}_{2}$ : El personal de la OTT es el factor clave para llevar a cabo los procesos de TT. A este respecto, en el estudio que reporta la OCDE en el 2003, se menciona que en la mayoría de los 
países de esta organización, las OTT operan con menos de cinco empleados de tiempo completo. Además, aunque en dicho estudio no se señalan cuáles son las competencias específicas que debe poseer el personal para conformar una OTT, se hace énfasis que dichas competencias deben de corresponder al ámbito de TT (OECD, 2003).

$\mathbf{P}_{3}$ : La gestión de la Propiedad Intelectual (PI) en una OTT es una de las actividades primordiales para impulsar el proceso de TT en una universidad. A este respecto, en el estudio que reporta la OCDE (2003), se menciona que a partir de la entrada en vigor de la Ley de Bay-Dole en 1980, las universidades de EE. UU. incrementaron considerablemente el número de patentes, estimulando así la creación de empresas (OECD, 2003).

$\mathbf{P}_{4}$ : El licenciamiento de tecnologías es otra opción que tiene una OTT para realizar actividades de transferencia de conocimiento generado dentro de las Universidades y CIs. A este respecto, Lockett, Wright y Franklin (2003) señalan que el licenciamiento de tecnología es la modalidad de transferencia de conocimiento que más utilizan las OTT actualmente.

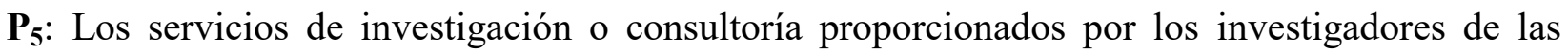
universidades o CIs, a través de las OTT a terceros, es otra forma de transferir el conocimiento. A este respecto, en el estudio que reporta la OCDE (2003) se menciona que la: "consultoría, es ofrecer al sector privado las habilidades y conocimientos de la comunidad académica para resolver problemas.” Asimismo, Perkmann y Walsh (2007) señalan que la consultoría, servicios de investigación y contratos de investigación son servicios pagados por clientes externos a la universidad.

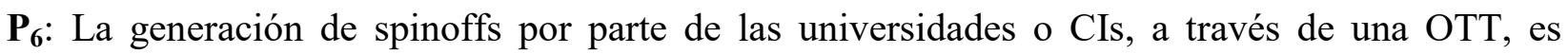
considerada otra alternativa para realizar TT. A este respecto, en el documento IDEA (2011) se señala que las spinoffs son unidades de negocios creadas por las IES o CI para comercializar una idea innovadora generada por los investigadores de estas entidades. 


\section{Unidad de análisis}

La unidad de análisis para esta investigación fue la OTT-BUAP, a través de la cual, los investigadores de dicha Universidad han solicitado 234 solicitudes de patente (OCT-PAT, 2019) en el periodo 2011-2018, de las cuales 47 ya han sido otorgadas, y solo una de ellas se ha comercializado a través de un licenciamiento. Esta oficina es coordinada, desde su establecimiento, por un único coordinador, quien trabajó por 12 años en el IMPI en diferentes cargos, dentro de ellos como examinador de patentes.

\section{Segundo paso - Preparación de la recolección de la información}

La recolección de la información proviene de fuentes primarias y secundarias; la información primaria se obtuvo de las entrevistas que se sostuvieron con personas de diferentes áreas que conforman la Dirección de Innovación y Transferencia de Conocimiento (DITCo) de la BUAP. En la Tabla 3 se muestra los datos de las personas que fueron entrevistadas.

Con respecto a la información proveniente de fuentes secundarias, se revisó la página web de la DITCo, específicamente el sitio de la OTT-BUAP. Ahí se encontraron, entre otros, diferentes lineamientos que regulan la operación de la OTT, así como evidencias de su portafolio de patentes disponibles al público para su comercialización, formatos de trabajo y algunos estudios realizados de vigilancia tecnológica. También, se consultaron algunas notas de prensa relacionadas con el desempeño de la OTT-BUAP.

\section{Tercer paso - Recolección de información.}

En cuanto a este punto, se realizaron entrevistas abiertas al grupo de personas mencionadas en el punto anterior. Es importante hacer mención que la transcripción de las entrevistas fue de carácter confidencial; por lo tanto, no se pudieron anexar a este documento.

\section{Cuarto paso - Análisis de la información.}

En esta sección se presentan los hallazgos encontrados durante las entrevistas realizadas a las personas que ostentan los puestos que se mencionan en la Tabla 3, así como las lecciones aprendidas y algunas recomendaciones que pudieran ser de utilidad para el especialista en TT. Dichos resultados se encuentran resumidos en las Tablas 4, 5, 6 y 7, los cuales corresponden a las proposiciones $\mathrm{P}_{1}, \mathrm{P}_{2}, \mathrm{P}_{3}$ y $\mathrm{P}_{4}$, respectivamente; mientras que los resultados para las proposiciones 
$\mathrm{P}_{5}$ y $\mathrm{P}_{6}$ no fueron resumidos en tablas, sino parafraseados, por no resultar relevantes para esta investigación. Es preciso señalar, que las lecciones y recomendaciones manifestadas en este documento no se pueden generalizar, puesto que surgen de un solo caso de estudio.

Tabla 3. Personas entrevistadas.

Table 3. People interviewed.

\begin{tabular}{|lc|}
\hline Puesto & Tiempo en ese puesto \\
\hline Coordinador de la OTT & 8 años \\
Responsable de la Unidad de Patentamiento de la OTT & 8 años \\
Responsable 1 de Evaluación Comercial de la OTT & 9 años \\
Responsable 2 de Evaluación Comercial de la OTT & 3 años 8 meses \\
Responsable de Evaluación Tecnológica de la OTT & 4 años 6 meses \\
Coordinador de Investigación Aplicada del Centro Universitario de & 11 años \\
Vinculación y Transferencia de Tecnología & 3 años \\
Director del CICE & 1 año \\
Técnico laboratorista de Makerzone & 3 años \\
Coordinador de Consultoría y Fábrica de Software & \\
\hline
\end{tabular}

Fuente: Elaboración propia.

Source: Own elaboration. 
Tabla 4 Análisis de las entrevistas realizadas acerca de la estructura organizacional de la OTT-BUAP $\left(\mathrm{P}_{1}\right)$.

Table 4 Analysis of the interviews conducted on the organizational structure of the OTT$\operatorname{BUAP}\left(\mathrm{P}_{1}\right)$.

Tópico

Estructura organizacional de la OTTBUAP

Barreras que han impedido al personal de la OTT-BUAP realizar actividades de TT

Modelo ideal de la estructura organizacional de la OTTBUAP

Solución del Conflicto laboral del personal que trabaja para la OTT y la Universidad

Observaciones y hallazgos de los autores

\section{Análisis de las entrevistas}

A este respecto, se identificó que a pesar de que los creadores de la OTT-BUAP conocían los tres tipos de estructura organizacional para el establecimiento de OTT que la OCDE (2003) reconoce viables para la TT, la estructura idónea para el establecimiento de la OTT-BUAP fue del tipo "Dedicada-interna", por las siguientes razones:

- La condición de estar establecida la OTT dentro de la universidad le permite a esta, fomentar la generación de una cultura en PI y TT en dicha comunidad.

- Además, la OTT aprovecha la infraestructura, capital humano e inversión de la misma universidad.

Derivado del análisis se encontró que las barreras principales podrían ser:

- La burocracia que existe en los procesos administrativos de la Universidad.

- La discontinuidad de los procesos de TT, debido a la rotación del personal de la parte directiva de la universidad, principalmente aquellos relacionados con la TT.

- La toma de decisiones, que en lo general, no depende del coordinador de la OTT, sino de otras jerarquías superiores dentro de la universidad.

- La implementación de una cultura de protección de la PI en la universidad.

Los entrevistados concuerdan que tal vez en México, el modelo ideal de la estructura organizacional de una OTT debiera ser la del tipo dedicada-externa. Dicho modelo evitaría, entre otras, la burocracia y toma de decisiones a las cuales están sujetas las OTT mexicanas del tipo dedicada-interna, principalmente, al depender del poder del Rector de la Universidad. Sin embargo, para lograr este tipo de independencia, las OTT deberían ser capaces de generar sus propios ingresos; nivel que la OTT-BUAP aún no ha alcanzado.

En contraste, en México solo existe una OTT del tipo dedicada-externa, la cual es la Operadora de Bienes y Servicios de la Universidad Politécnica de Pachuca, S.C.; pero, se desconocen hasta la fecha sus casos de éxito.

A este respecto, se encontró que los empleados de la OTT-BUAP no presentan dicho conflicto, debido a que ellos fueron contratados, exclusivamente, para atender procesos relacionados con la OTT-BUAP. Sin embargo, si en un futuro se llegara a necesitar que dicho personal tuviera que desempeñar actividades docentes en la universidad, el conflicto se subsanaría con el desarrollo de lineamientos que estipulen claramente la carga y plan de trabajo del empleado.

De fuentes secundarias de información, se identificó que la misión y visión de la BUAP no está alineada con la de la OTT-BUAP en el contexto de TT; a este respecto, se encontró que esta situación se debe a que el concepto y proceso de TT es reciente en México, el cual surge con la aparición de las OTT en el 2011.

En otro contexto, si bien es cierto que existen en el mundo OTT exitosas del tipo dedicada-externa tales como la de la Universidad de Oxford y la Universidad Hebrea de Jerusalén (Meseri y Maital, 2001), por mencionar algunas; en México, el contexto es diferente para que las OTT del tipo dedicada-interna evolucionen al tipo dedicada-externa. Por último, se confirma $\mathrm{P}_{1}$, debido a que la OTT-BUAP cumple con la recomendación que hace la OCDE, al tener una estructura organizacional del tipo dedicada-interna para su funcionamiento.

Fuente: Elaboración propia.

Source: Own elaboration.

No 24, Vol. 12 (1), 2020. ISSN 2007 - 0705, pp.: 1 - 31

- 14 - 
Las lecciones aprendidas acerca del proceso del establecimiento de la estructura organizacional de la OTT-BUAP, fueron las siguientes:

1. El establecimiento de una OTT es un proceso que debe ser considerado dentro de la planeación estratégica de la universidad; y por ende, ser parte fundamental en la misión y visión de la propia institución.

2. En un inicio, la estructura organizacional de una OTT universitaria debe ser del tipo "Dedicada-interna", para fomentar una cultura en la protección de la PI y TT en dicha comunidad universitaria, y aprovechar la infraestructura, capital humano e inversión de la misma universidad.

Las recomendaciones para el proceso del establecimiento de la estructura organizacional de una OTT en México son las siguientes:

1. Es importante que la misión y visión de aquellas Universidades o CIs que se dedican a realizar actividades de TT, sean modificadas en el ámbito de emprendimiento y TT. Y además la misión y la visión, tanto de la universidad como su OTT, deban de estar alineadas para ser más eficientes.

2. Se sugiere que la estructura organizacional inicial de una OTT sea del tipo dedicada interna, para que pueda sobrevivir los primeros años, pero una vez que dicha oficina sea financieramente auto sostenible, entonces, se recomienda que su estructura organizacional sea migrada al tipo dedicada externa para operar de manera más eficiente. 
Tabla 5. Análisis de las entrevistas realizadas acerca del personal que conforma la OTT$\operatorname{BUAP}\left(\mathrm{P}_{2}\right)$.

Table 5. Analysis of the interviews applied to the personnel that are part of the OTT-BUAP $\left(\mathrm{P}_{2}\right)$.

Tópico

¿Por qué la OTT-BUAP opera con personal externo a la universidad?

¿Cuáles son los perfiles idóneos que deben de tener los empleados de una OTT?

¿Cómo sufraga los salarios de su personal la OTT-BUAP?

¿Cómo es capacitado el personal de la OTT-BUAP en tópicos de TT?

Observaciones y hallazgos de los autores

\section{Análisis de las entrevistas}

A este respecto, la mayoría de los entrevistados coinciden que es muy raro, al menos en México, encontrar en las universidades públicas individuos con las capacidades para realizar actividades relacionadas con la TT. Ello es debido, principalmente, a que las universidades mexicanas no fueron concebidas para la comercialización de la ciencia y tecnología; razón por la cual, los perfiles idóneos para la operación de la OTT deben ser buscados fuera de la universidad. Además, a estas personas se les debe proporcionar la capacitación apropiada, porque el proceso de la TT es muy vasto y complejo.

En este contexto, se encontró que la OTT-BUAP esta conformada por un equipo de cinco personas, un abogado especializado en propiedad intelectual, tres tecnólogos en diferentes áreas de conocimiento, y un experto en TT para la gestión de la OTT quien trabajo por 12 años en el IMPI ocupando varios puestos, dentro de ellos, el de examinador de patentes. Es importante mencionar, que se encontró que las cinco personas, además de cumplir con funciones específicas para cada uno de los puestos de gestión de la OTT, se han especializado en la redacción de patentes en diferentes áreas de conocimiento para atender la demanda de los investigadores.

En relación con este punto, los entrevistados concuerdan que la OTT-BUAP debería ser auto sostenible a través de las entradas económicas que podrían ser generadas por los licenciamientos y servicios de consultoría ofertados, o la creación de spinoffs. Sin embargo, como hasta la fecha la OTT-BUAP no ha madurado en esos procesos de TT, la BUAP paga los salarios de los empleados de la OTT. Actualmente, esta oficina cuenta con cinco personas, dos de ellas reciben sus salarios vía nomina, puesto que son empleados de base de la misma universidad, mientras que los otros tres están siendo contratados por honorarios.

Por lo que respecta a este punto, se encontró que el personal ha recibido capacitación a través de la asistencia a diplomados y congresos nacionales e internacionales relacionados con la transferencia y comercialización de tecnología, asistiendo a los cursos y talleres que el IMPI oferta al público en general. Además, los entrevistados destacan que los cursos que más les han ayudado para la redacción de patentes y temas relacionados con la protección de la PI han sido los que oferta en línea la Organización Mundial de la Propiedad Intelectual(WIPO, 2019).

Se identificó que para detonar la TT en la BUAP, se tuvo que contratar personal para ser capacitado en tópicos del ámbito de PI y TT, porque la BUAP no contaba con esos perfiles profesionales. Además, se halló que la OTT-BUAP está funcionando con cinco empleados, los cuales han desarrollado capacidades en el área de PI y TT, lo cual concuerda, en parte, con lo que se menciona en el reporte de la OCDE (2003), donde la mayoría de las OTT de los países que conforman dicha organización funcionan con menos de 5 empleados con competencias en el ámbito de TT. Por lo consiguiente se confirmó $\mathrm{P}_{2}$.

Fuente: Elaboración propia.

Source: Own elaboration.

$\mathrm{N}^{\mathrm{o}}$ 24, Vol. 12 (1), 2020. ISSN 2007 - 0705, pp.: 1 - 31

-16 - 
Las lecciones aprendidas acerca del proceso de contratación del personal que conforma la OTTBUAP, fueron las siguientes:

1. Contratar un especialista en TT o PI para articular y gestionar la OTT, siempre y cuando, el presupuesto y la normativa de la universidad lo permita.

2. Para que una OTT sea operable de manera eficiente, se necesita que cuente al menos con los siguientes perfiles: un abogado especializado en propiedad intelectual, tecnólogos en diferentes ramas de ingeniería, y un experto en TT o equivalente para administrar la OTT.

Las recomendaciones para el proceso de la contratación del personal de una OTT en México, fueron las siguientes:

1. Se recomienda contratar o capacitar personal idóneo y con experiencia para establecer y operar una OTT profesionalizada. Dicho personal debe ser capaz de diseñar políticas institucionales de operación eficiente para atender la producción de conocimiento y tecnología generada por la comunidad de la universidad.

2. Se sugiere que el personal de la OTT se capacite en diferentes tópicos que comprende la TT. Por ejemplo, la redacción de patentes, vigilancia tecnológica, comercialización de tecnología, evaluación de tecnologías, licenciamiento, solo por mencionar algunos de ellos.

3. Es aconsejable que la OTT empiece a generar ingresos, no solo de aquellos intangibles generados por la comunidad universitaria, sino también, por aquellos servicios que pudiera ofrecer la OTT al exterior. Ejemplos de tales servicios pueden ser la redacción de patentes a externos a la universidad, consultoría en vigilancia tecnología y tramite de marcas, por mencionar algunos. 
Tabla 6. Análisis de las entrevistas realizadas acerca de la gestión de la PI en la OTT-BUAP $\left(\mathrm{P}_{3}\right)$.

Table 6. Analysis of interviews conducted about Intellectual Property management in the

\begin{tabular}{|c|c|}
\hline Tópico & Análisis de las entrevistas \\
\hline $\begin{array}{l}\text { ¿Cómo ha } \\
\text { logrado la } \\
\text { OTT-BUAP } \\
\text { establecer una } \\
\text { cultura de } \\
\text { protección de } \\
\text { PI dentro de la } \\
\text { universidad? }\end{array}$ & $\begin{array}{l}\text { - A través de conferencias sobre TT y PI impartidas por el personal de la OTT a } \\
\text { la comunidad universitaria. } \\
\text { - Instauración del día de la patente en la BUAP, en donde los inventores } \\
\text { exponen sus invenciones. } \\
\text { - Por medio del establecimiento de una ceremonia oficial presenciada por el } \\
\text { rector de la BUAP para reconocer ante la comunidad universitaria a aquellos } \\
\text { investigadores que logran obtener una patente. } \\
\text { - Por medio de entrevistas hechas por personal del CONACyT a los inventores } \\
\text { de la BUAP, las cuales son difundidas a nivel nacional por el CONACyT. } \\
\text { - A través de la creación de la revista Alianzas y Tendencias BUAP en donde se } \\
\text { publican temas relacionados con la transferencia y comercialización de la } \\
\text { tecnología producida en dicha universidad. }\end{array}$ \\
\hline
\end{tabular}

¿Cómo ha motivado la OTT-BUAP a los investigadores a proteger los resultados de sus investigaciones?

¿Cómo hizo la OTT-BUAP para incrementar el número de patentes generadas por sus investigadores?

¿Cómo beneficia a la BUAP el incremento de patentes que han generado sus investigadores durante los últimos años?

Observaciones y hallazgos de los autores
- Quitando la responsabilidad de la redacción de patente a los investigadores; es decir, el personal de la OTT se encarga del proceso de redacción de patentes.

- Pagando al 100\% los derechos de la gestión de la patente ante el IMPI; es decir, la BUAP absorbe todos los gastos que conlleva este proceso.

- Considerar las solicitudes de patente dentro de productos a evaluar en la beca al estímulo del desempeño docente.

- Otorgando al investigador el $30 \%$ de las regalías generadas de un posible licenciamiento de su patente (CUVYTT, 2014).

La estrategia que se identificó, fue la conformación de un equipo de trabajo multidisciplinario especializado en la redacción de patentes, apoyado en un plan de incentivos estratégico para motivar a los investigadores a proteger los resultados de sus investigaciones. Este equipo de trabajo, además de transformar en solicitudes de patente aquellos resultados de las investigaciones hechas por los investigadores de la BUAP, también, realiza trabajos de vigilancia tecnológica, estudios de libertad de operación de patentes, desarrollo de planes y modelos de negocios, entre otros. También se encontró que la BUAP tiene un reglamento donde se establecen de forma clara los beneficios y regalías de la comercialización y TT de los intangibles, así como la delimitación de los derechos morales y patrimoniales de los inventores (CUVYTT, 2014).

El principal beneficio es prestigio para la BUAP, ya que actualmente tanto las solicitudes de patentes como la obtención de ellas, son indicadores para evaluar a las universidades a nivel nacional. Además, el incremento de patentes habilita a los investigadores/inventores a formar cuerpos académicos capaces de hacerse acreedores a recursos financieros para continuar con sus investigaciones; trayendo como resultado, el desarrollo de nuevas capacidades y habilidades en los investigadores que se podrían ver reflejadas en la enseñanza a sus alumnos.

Se encontró que la OTT-BUAP cuenta con un plan estratégico de incentivos para motivar a los investigadores a proteger los resultados de sus investigaciones, respaldado por unos lineamientos que regulan la protección de la PI, todo esto con el objetivo específico de incrementar el indicador de patentes en dicha universidad. Por lo tanto, $\mathrm{P}_{3}$ se confirma.

Fuente: Elaboración propia.

Source: Own elaboration. 
Las lecciones aprendidas del proceso de gestión de la PI en la OTT-BUAP, fueron las siguientes:

1. Es importante contar con un reglamento donde se estipule de manera ordenada y equitativa los beneficios y regalías de la comercialización y TT de los intangibles, así como el establecimiento claro de los derechos morales y patrimoniales de los inventores.

2. Contar con un plan de estratégico de incentivos para motivar a los investigadores a fomentar y proteger los resultados de sus investigaciones.

3. Establecer un equipo de trabajo multidisciplinario especializado en la redacción de patentes, vigilancia tecnológica, estudios de libertad de operación de patentes, desarrollo de planes y modelos de negocios, entre otros.

Las recomendaciones para el proceso de gestión de la PI de una OTT en México, son las siguientes:

1. Se recomienda que la parte directiva de la universidad junto con el personal de la OTT, implementen una política interna donde se establezca los lineamientos relacionados con la protección de la PI que coadyuve a fomentar una cultura de la protección de PI y TT en la comunidad universitaria.

2. Se sugiere el establecimiento de un plan estratégico de incentivos que motive a la comunidad universitaria a proteger los resultados de sus investigaciones.

3. Se aconseja conformar un equipo de trabajo multidisciplinario experto en las competencias de TT para gestionar y operar una OTT. 
Tabla 7. Análisis de las entrevistas realizadas acerca de los licenciamientos hechos por la OTT-BUAP $\left(\mathrm{P}_{4}\right)$.

Table 7. Analysis of the interviews conducted about the licensing done by the OTT-BUAP

$\left(\mathrm{P}_{4}\right)$.

\begin{tabular}{|c|c|}
\hline Tópico & Análisis de las entrevistas \\
\hline $\begin{array}{l}\text { ¿Cómo ha } \\
\text { logrado, la } \\
\text { OTT-BUAP, } \\
\text { licenciar sus } \\
\text { tecnologías? }\end{array}$ & $\begin{array}{l}\text { A este respecto, se encontró que hasta la fecha el personal de la OTT-BUAP, solo } \\
\text { ha podido licenciar una tecnología relacionada con un fármaco para tratar la } \\
\text { enfermedad del pie diabético. También se halló que algunas de las estrategias } \\
\text { utilizadas para impulsar el proceso de licenciamiento fueron: } \\
\text { - Oferta de un portafolio de } 38 \text { de tecnologías para su comercialización a través } \\
\text { de su página Web de la OTT-BUAP (OCT-BUAP, 2019). } \\
\text { - Realizando estudios de vigilancia tecnológica, } \\
\text { - Búsqueda directa de clientes. } \\
\text { - A través de la divulgación de la revista Alianzas y Tendencias BUAP. }\end{array}$ \\
\hline $\begin{array}{l}\text { ¿Cómo } \\
\text { interviene, un } \\
\text { investigador de } \\
\text { la BUAP, en el } \\
\text { proceso de } \\
\text { licenciamiento } \\
\text { de su } \\
\text { invención? }\end{array}$ & $\begin{array}{l}\text { A este respecto se encontró que todas las invenciones que se han protegido ante el } \\
\text { IMPI via la OTT-BUAP han sido generadas dentro de la BUAP, por lo tanto, le } \\
\text { pertenecen a la misma, sin embargo, los derechos morales se les otorgan a los } \\
\text { inventores. Por lo anterior, el investigador no interviene en el licenciamiento, ya } \\
\text { que la OTT es la que se encarga de realizar la redacción de la patente y la } \\
\text { comercializacion o transferencia de la tecnología. }\end{array}$ \\
\hline $\begin{array}{l}\text { ¿Cómo son } \\
\text { distribuidos, los } \\
\text { dividendos } \\
\text { obtenidos por } \\
\text { los } \\
\text { licenciamientos? }\end{array}$ & $\begin{array}{l}\text { A este respecto, se encontró que las regalías obtenidas de un licenciamiento son } \\
\text { distribuidas de acuerdo con los lineamientos correspondientes del CUVYTT } \\
\text { (2014) de la siguiente forma: } \\
\text { - El } 30 \% \text { de las regalías se le comparten al investigador/inventor de la invención. } \\
\text { - El } 30 \% \text { para la adquisición de materiales y equipo para el grupo de } \\
\text { investigadores o autores que generó la invención. } \\
\text { - Y el } 40 \% \text { para la OTT, lo cual es utilizado para cubrir los gastos de protección } \\
\text { de propiedad intelectual, gastos para estudios de mercado y elaboración de plan } \\
\text { de negocios, y pago de honorarios a los empleados de la OTT. }\end{array}$ \\
\hline $\begin{array}{l}\text { ¿Cómo impacta } \\
\text { el } \\
\text { licenciamiento } \\
\text { de alguna } \\
\text { tecnología a la } \\
\text { comunidad } \\
\text { universitaria? }\end{array}$ & $\begin{array}{l}\text { - El licenciamiento de una tecnología impacta directamente en el beneficio } \\
\text { económico del investigador, motivándolo al desarrollo de más tecnologías que } \\
\text { tengan impacto en la sociedad. } \\
\text { - Los dividendos obtenidos por la modalidad de licenciamiento también } \\
\text { benefician a la comunidad universitaria, en el mejoramiento de la } \\
\text { infraestructura y equipamiento de laboratorios para el desarrollo de nuevas } \\
\text { tecnologías. }\end{array}$ \\
\hline $\begin{array}{l}\text { Observaciones y } \\
\text { hallazgos de los } \\
\text { autores }\end{array}$ & $\begin{array}{l}\text { Aunque se encontró que la OTT-BUAP tiene en su catálogo de licenciamiento de } \\
\text { patentes } 38 \text { tecnologías para su licenciamiento (OCT-BUAP, 2019), la mayoría de } \\
\text { ellas no tienen un segmento de mercado identificado, ya sea, porque la invención } \\
\text { no es comprendida por el mercado; o bien, por la necesidad de un estudio de } \\
\text { mercado previo al desarrollo de la tecnología, tal y como lo recomiendan Blank y } \\
\text { Dorf (2012). } \\
\text { Este hallazgo, en general, contradice lo que señalan Lockett, Wright y Franklin } \\
\text { (2003) acerca de que el licenciamiento de tecnología es la modalidad de } \\
\text { transferencia que más utilizan las OTT. Por lo consiguiente, } \mathrm{P}_{4} \text { no se confirma. }\end{array}$ \\
\hline
\end{tabular}

Fuente: Elaboración propia.

Source: Own elaboration. 
Las lecciones aprendidas del proceso de licenciamiento de la OTT-BUAP, fueron las siguientes:

1. Los trabajos de investigación deben de estar enfocados a resolver necesidades que demanda el mercado, siempre y cuando se desee patentar para comercializar o transferir alguna tecnología.

2. El tener intangibles protegidos para su comercialización no es suficiente, es necesario que dichos intangibles deban de contar con aquellas pruebas, trámites complementarios, planes y modelos de negocios que hagan la tecnología más asequible al mercado.

3. Tener un reglamento interno en el contexto de protección de la PI y TT donde se estipulen de manera equitativa la distribución de las regalías entre la universidad e inventores, incrementa la confianza y motivación de los investigadores de la universidad.

Las recomendaciones para el proceso de licenciamiento que pueda ser establecido en cualquier OTT en México, fueron las siguientes:

1. Se recomienda que el personal de la OTT realice trabajos de vigilancia tecnológica en diferentes áreas del conocimiento que sean de interés para ser compartidos con los investigadores de la comunidad universitaria, para impulsar el desarrollo de innovaciones que tengan un mercado identificado.

2. Desarrollar una estrategia de publicidad y marketing que permita ofertar los intangibles de la universidad para su comercialización.

3. Se sugiere que se hagan estudios de mercado para analizar el potencial de la tecnología y con ello tomar la decisión de su patentamiento, tal y como lo recomiendan Blank y Dorf (2012).

\section{Análisis de las entrevistas realizadas acerca de la prestación de servicios de consultoría proporcionados por los investigadores de la BUAP vía la OTT-BUAP $\left(\mathbf{P}_{5}\right)$}

A este respecto, aunque se encontró un padrón de consultores conformado por investigadores de la BUAP (OCT-PAD, 2018), no se hallaron registros de servicios de consultoría prestados directamente por dichos investigadores hacia otras organizaciones. Esto se debe en gran medida, desde el punto de vista de algunos de los entrevistados, a la carga académica a la que están 
sujetos los investigadores; lo cual concuerda en parte con lo que se menciona en IDEA (2011), que el proceso de consultoría en algunas universidades no forma parte de las actividades de la OTT, debido a la dificultad organizacional que conlleva este proceso.

Por otra lado, de acuerdo al padrón de beneficiados del Programa de Estímulos a la Innovación (PEI) emitido por el CONACyT en el 2017, donde dicho programa busca fomentar la vinculación de la Industria-Gobierno-Universidad para el desarrollo de innovaciones tecnológicas, se encontró que la BUAP ha participado en 37 proyectos de desarrollo tecnológico con diferentes empresas durante el periodo 2009-2017, involucrando varios de sus investigadores (PEI, 2017). Sin embargo, aunque dicho programa generó ingresos económicos a la BUAP debido a la participación directa de algunos de sus investigadores, dichos ingresos no pudieron ser clasificados como ingresos por servicios de consultoría; porque el PEI es un programa del gobierno federal mexicano. Por lo tanto, $\mathrm{P}_{5}$ no se confirma.

Las lecciones aprendidas del proceso de los servicios de consultoría proporcionados por los investigadores de la BUAP vía la OTT-BUAP, fueron las siguientes:

1. Aunque la OTT-BUAP ha conseguido establecer un padrón de consultores a través de diferentes estrategías basada en incentivos, esto no ha coadyuvado a detonar que los servicios de consultoria.

2. Dentro de las modalidades para realizar TT, la consultoría es uno de los procesos más difíciles de llevar a cabo dentro de una universidad debido a que esa actividad se encuentra muy distante del perfil del investigador; es decir, el investigador fue contratado por la universidad para impartir enseñanza y hacer investigación, más no para prestar servicios de consultoría.

Las recomendaciones para el proceso de los servicios de consultoría para una OTT en México, fueron las siguientes:

1. Se recomienda que se busquen alternativas para incentivar al investigador a proporcionar servicios de consultoría. 
2. Se aconseja que las universidades revisen sus reglamentos de trabajo interno para que las cargas académicas de los investigadores sean coherentes con sus responsabilidades académicas y actividades relacionadas con la TT.

\section{Análisis de las entrevistas realizadas acerca del proceso de creación de spinoffs o spinouts por la OTT-BUAP $\left(\mathbf{P}_{6}\right)$}

Aunque se hallaron dos Estudios de viabilidad para la creación de empresas spin-off, uno para inoculantes multiespecies (Monge, Leal, Sánchez y De la Cerrna, 2018b), y otro para energías limpias-biodiesel (Monge, Leal, Sánchez y De la Cerrna, 2018); estos nunca fueron llevados a cabo por falta de emprendedores.

Por otro lado, aunque la BUAP cuenta con un ecosistema de innovación conformado principalmente por una masa crítica de investigadores, un centro de incubación y una OTT, no se encontró la creación de alguna spinoff o spinout universitaria de base tecnológica por medio de la OTT-BUAP. Por lo consiguiente, $\mathrm{P}_{6}$ no se confirma.

Las lecciones aprendidas del proceso de creación de spinoffs o spinouts de la OTTBUAP, fueron las siguientes:

1. De las tres modalidades que tiene una OTT para realizar actividades de TT, las cuales son consultoría, licenciamiento y creación de spinoffs, la última es una de las más difíciles. Esto se podría deber en gran medida tal y como le señalan Nikulin et al. (2017), a que el emprendimiento universitario implica adquirir más habilidades, responsabilidades y riesgos, que muchos de los investigadores no tienen, ni están dispuestos a adquirir por miedo a la incertidumbre que conlleva establecer una empresa.

Las recomendaciones para el proceso de creación de spinoffs o spinouts para una OTT de México, fueron las siguientes:

1. Se recomienda que se busquen alternativas para incentivar a los investigadores a crear empresas de base tecnológica.

2. Se aconseja que las universidades o CIs que realicen actividades de TT, revisen sus reglamentos de trabajo interno para que las prestaciones, como la de año sabático o 
Desempeño de una Oficina de Transferencia de Tecnología en el contexto de gestión de patentes: Estudio de caso de la OTT de la Benemérita Universidad Autónoma de Puebla

similares, que tienen algunos investigadores sean ocupadas para la creación de empresas de base tecnológica siempre y cuando, los investigadores deseen emprender.

\section{Paso 5 - Informe}

Finalmente, este trabajo integra toda la descripción del caso exploratorio y descriptivo que se estructuró en los cinco apartados que conforman este documento. Adicionalmente, la validez del caso se detalla a continuación.

\section{Validez del caso}

Para demostrar la validez, confiabilidad y consistencia de un caso de estudio, Yin (1994) propone ciertas pruebas (Tabla 8), las cuales se han adaptado para validar este estudio de caso.

Tabla 8. Validez del caso.

Table 8. Validity of the case.

\begin{tabular}{|c|c|c|}
\hline Prueba & Estrategia & Fase \\
\hline Validez constructiva & $\begin{array}{l}\text { Referentes conceptuales y teóricos sobre la TT, } \\
\text { licenciamiento, patentamiento, consultoría y spin- } \\
\text { off, especialmente para el caso de una OTT. } \\
\text { Entrevistas abiertas para la recolección de } \\
\text { información, que es un método ampliamente } \\
\text { validado. Además, información secundaria de } \\
\text { fuentes oficiales de la BUAP como los estatutos, } \\
\text { reglamentos de propiedad intelectual y los sitios } \\
\text { oficiales de la OTT-BUAP. }\end{array}$ & Revisión de la literatura \\
\hline Validez interna & $\begin{array}{l}\text { Comparación sistemática de la literatura con los } \\
\text { procesos de licenciamiento, patentamiento y } \\
\text { consultoría de la OTT-BUAP. }\end{array}$ & Metodología. \\
\hline & $\begin{array}{l}\text { Elección de temas y subtemas para la realización } \\
\text { de las entrevistas estructuradas basados, } \\
\text { principalmente, en las documentos de IDEA, } \\
\text { 2011; Manderieux, 2011; y SE-CONACyT } \\
\text { (2012). }\end{array}$ & $\begin{array}{l}\text { Apartado, criterios para } \\
\text { el análisis de la } \\
\text { información. }\end{array}$ \\
\hline Validez externa & $\begin{array}{l}\text { Uso de literatura internacional de amplia } \\
\text { aceptación. } \\
\text { Consulta en bases de datos especializadas. } \\
\text { La selección de una unidad de análisis (OTT- } \\
\text { BUAP). }\end{array}$ & $\begin{array}{l}\text { Apartado, unidad de } \\
\text { análisis. }\end{array}$ \\
\hline Fiabilidad & $\begin{array}{l}\text { Se aplicó rigurosamente el método del estudio del } \\
\text { caso tal como se propone Yin (1994). Además la } \\
\text { recolección de la información por medio de las } \\
\text { entrevistas se estructuró de acuerdo a las } \\
\text { proposiciones extraídas de la literatura } \\
\text { especializada. Las entrevistas fueron aplicadas a } \\
\text { personas especialistas en la materia. }\end{array}$ & Metodología. \\
\hline
\end{tabular}

Fuente: Elaboración propia.

Source: Own elaboration. 


\section{Conclusiones}

El propósito de esta investigación fue aplicar el método del estudio del caso a la unidad de análisis OTT-BUAP, basándose en seis proposiciones obtenidas de tres fuentes secundarias para dar respuesta a la pregunta de investigación: ¿Cómo ha logrado la OTT-BUAP posicionarse dentro de las mejores OTT en México, en el ámbito de gestión de patentes, durante el periodo 2011-2018?

Según los resultados encontrados, se concluye que la estrategia que aplicó la OTT-BUAP para posicionarse dentro las mejores OTT en México en el contexto de gestión de patentes, consistió en tres momentos que se describen a continuación.

Primero, el establecimiento de una estructura organizacional de OTT del tipo dedicada-interna para aprovechar la infraestructura, capital humano e inversión económica de la misma universidad para la realización de tareas relacionadas con la protección de la PI y TT (Confirmación de $\mathrm{P}_{1}$ ).

Segundo, la conformación de un equipo de trabajo multidisciplinario, integrado, por personal ajeno a la BUAP entrenado en el área de protección de PI y TT para generar políticas institucionales eficientes para atender las diferentes necesidades de protección intelectual y comercialización de tecnologías que demanda la comunidad de la BUAP. Siendo el factor determinante de éxito, el haber contratado a un investigador proveniente del IMPI, experto en la redacción de patentes y TT, para gestionar la OTT-BUAP desde su creación hasta la fecha (Confirmación de $\mathrm{P}_{2}$ ).

Tercero, el establecimiento de un plan estratégico de incentivos para motivar a los investigadores a proteger los resultados de sus investigaciones, respaldado por unos lineamientos que regulan la protección de la PI (Confirmación de $\mathrm{P}_{3}$ ).

Dentro de las oportunidades de mejora que se encontraron durante el desarrollo de esta investigación y que están asociadas a las no confirmaciones de $\mathrm{P}_{4}, \mathrm{P}_{5}$ y $\mathrm{P}_{6}$ fueron las siguientes.

Desarrollar y aplicar un proceso de licenciamiento eficaz para transferir el portafolio de intangibles a otras organizaciones para su comercialización.

Buscar otros incentivos u estrategias que motiven a los investigadores prestar servicios de consultoría.

Diseñar estrategias que permitan ir evaluando por etapas el desarrollo de las investigaciones hechas por los investigadores, para detectar a tiempo la factibilidad de 
transferencia o comercialización de dichas tecnologías a través de licenciamientos o la creación de spinoffs respectivamente.

Es importante hacer mención que derivado de los resultados obtenidos casi nulos en los procesos de consultoría, licenciamiento y creación de spinoffs (No confirmaciones de $\mathrm{P}_{4}, \mathrm{P}_{5}$, y $\mathrm{P}_{6}$ respectivamente), el ecosistema de innovación de la BUAP conformado por su centro de incubación, OTT, área de Investigación Aplicada, Makerzone y la Fábrica de Software, se está reestructurando para hacer más eficientes dichos procesos. Esta reestructuración podría ser la clave para convertir a la BUAP en una universidad emprendedora y líder en el campo de la TT en México.

Es importante mencionar que la implicación que podría traer a un gestor de una OTT de México o cualquier parte del mundo, el no asegurar la proteccion de la PI dentro de una universidad, es el riesgo de no tener intangibles para su transferencia o comercialización a través de licenciamientos o creación de spinoffs.

Finalmente, en el contexto de trabajos futuros, se recomiendan hacer estudios similares en OTT mexicanas que posean una estructura organizacional del tipo "dedicada-externa", con el objeto de hacer contrastes con aquellas que posean una estructura "dedicada-interna" que nos permitan medir el desempeño entre ambos tipos de OTT, y de esta forma, poder encontrar un modelo de TT que sirva de referencia para las OTT mexicanas.

\section{$\underline{\text { Reconocimientos }}$}

Este trabajo de investigación fue apoyado por una beca de investigación del Programa para el Desarrollo Profesional Docente (PRODEP), y el Tecnológico Nacional de México/Instituto Tecnológico de Apizaco (Núm. De Folio: ITAPI-010).

Se agradece a la Coordinación de la Oficina de Comercialización de Tecnología de la BUAP por darnos la oportunidad de haber llevado a cabo esta investigación en sus instalaciones, y además, permitirnos divulgar este conocimiento en el contexto académico y científico.

\section{$\underline{\text { Referencias }}$}

Alvarado Moreno, F. (2018). El Papel de las Oficinas de Transferencia Tecnológica (OTT) en las Universidades: Una Perspectiva de la Última Década. Journal of technology management \& innovation, 13(3), 104-112. http://dx.doi.org/10.4067/S0718-27242018000300104 
Arundeep, P. (2016). The Evolution of Technology Transfer. Recuperado el 25/09/2019, de https://www.linkedin.com/pulse/evolution-technology-transfer-arundeep-s-pradhan-rttp

Monge, B., Leal, J., Sánchez, G. y De la Cerna, K. (2018). Plan de empresa spin-off de energías limpias-biodisel. Recuperado el 26/06/2019, de http://www.ditco.buap.mx/recursos/documentos/spin-off-biodiesel.pdf

Monge, B., Leal, J., Sánchez, G. y De la Cerna, K. (2018b). Plan de empresa spin-off de inoculante multiespecies. Recuperado el 26/06/2019, de http://www.ditco.buap.mx/recursos/documentos/spin-off-inoculante.pdf

Blank, S. y Dorf, B. (2012). The startup owner's manual: The step-by-step guide for building a great company. BookBaby.

BUAP (2018). 1er. Informe de actividades del Rector J. Alfonso Esparza Ortíz. Recuperado el 23 de febrero de 2019, de https://www.pdi.buap.mx/sites/default/files/pdf/1er_informe_2018/informe.pdf

CII. (2011). Programa Nacional de Innovación. México, DF: Comité Intersectorial para la Innovación.

CONACyT (2018). Oficinas de Transferencia de Tecnología (OTT) reconocidas por PROSOFTinnovación, Fondo Sectorial de Innovación (antes Fondo Sectorial de Innovación Secretaría de Economía-CONACyT). Recuperado el 30 de enero de 2019, de https://www.conacyt.gob.mx/index.php/sni/convovatorias-programa-de-estimulos-a-lainnovacion/convocatoria-2018-pei/14658-directorio-ot/file

CORFO. (2016). Transferencia tecnológica en Chile. Estudio de caracterizazión de las actividades y resultados de los procesos de transferencia de tecnológica desde los centros de conocimiento en Chile. Corporación de Fomento de la Producción. Recuperado el 26/09/2019, de https://www.corfo.cl/sites/Satellite?blobcol=urldata\&blobkey=id\&blobtable=MungoBlob s\&blobwhere $=1475166876180 \&$ ssbinary $=$ true

Cruz Novoa, A. (2016). Innovación de Base Científica-Tecnológica desde las Universidades de Iberoamérica. Journal of technology management \& innovation, 11(4), 1-4. http://dx.doi.org/10.4067/S0718-27242016000400001

CUVYTT. (2014). Lineamientos para el funcionamiento y operación del Centro de Vinculación y Transferencia de Tecnología de la Benemérita Universidad Autónoma de Puebla. 
Desempeño de una Oficina de Transferencia de Tecnología en el contexto de gestión de patentes: Estudio de caso de la OTT de la Benemérita Universidad Autónoma de Puebla

Recuperado el 23/09/2019, de http://www.ditco.buap.mx/recursos/documentos/cuvyttLineamientosFuncionamientoOperacion.pdf

Dos Santos, M. E. R., \& Torkomian, A. L. V. (2013). Technology transfer and innovation: The role of the Brazilian TTOs. International Journal of Technology Management \& Sustainable Development, 12(1), 89-111.

Estrada González, G. A. (2009). Creación de oficinas de transferencia de tecnología (OTTs) como mecanismos de impulso de la transferencia tecnológica en los Centros de Investigación CONACYT. México: Instituto Politécnico Nacional.

Gotkin, J. (2012). The United States Bayh-Dole Act and Its Effect on University Technology Transfer. Nomos Verlagsgesellschaft mbH \& Co. KG.

Granieri, M. y Basso, A. (2019). Building Capacity Building in Technology Transfer. An Introduction. In Capacity Building in Technology Transfer (pp. 1-6). Springer, Cham.

Heraldo (2018). BUAP tiene el tercer lugar a nivel nacional en creación de patentes. Recuperado el 20 de abril de 2019, de https://heraldodemexico.com.mx/estados/buap-tiene-el-tercerlugabuap-tiene-el-tercer-lugar-a-nivel-nacional-en-creacion-de-patentesr-a-nivel-nacionalen-la-creacion-de-patentes/

IDEA (2011). La transferencia de conocimiento. Mejores prácticas internacionales para el diseño de un programa de transferencia de conocimiento en México. Recuperado el 1 de febrero de 2019, de https://website-c230consultores.nyc3.digitaloceanspaces.com/IDEA/files/EstrategiaTransferenciaDeConocimi entoSEconacytVf_1536351040.pdf

IMPI (2018). Conoce las universidades mexicanas que más patentan. Recuperado el 18 de mayo de 2019, de https://www.gob.mx/impi/articulos/conoce-las-universidades-mexicanas-quemas-patentan? idiom $=\mathrm{es}$

Lockett, A., Wright, M. y Franklin, S. (2003). Technology transfer and universities' spin-out strategies. Small Business Economics, 20(2), 185-200.

Manderieux, L. (2011). Guía práctica para la creación y la gestión de oficinas de transferencia de tecnología en universidades y centros de investigación de América Latina. El rol de la propiedad intelectual. Organización Mundial de la Propiedad Intelectual.

Medellín Molina, E. R. (2015). Diagnóstico de las oficinas de transferencia de conocimiento OTC en México. 
Mendoza Rojas, J. (2018). Subsistemas de Educación Superior. Estadística básica 20062017. Cuadernos de Trabajo de la Dirección General de Evaluación Institucional, ano, 5.

Meseri, O. \& Maital, S. (2001). A survey analysis of university-technology transfer in Israel: evaluation of projects and determinants of success. The Journal of Technology Transfer, 26(1-2), 115-125.

Nikulin Chandia, C., Viveros Gunckel, P., Dorochesi Fernandois, M., Crespo Márquez, A., \& Lay Bobadilla, P. (2017). Metodología para el análisis de problemas y limitaciones en emprendimientos universitarios. Innovar, 27(63), 91-105.

OCT-BUAP (2019). Licenciamiento de patentes. Recuperado el 3 de mayo de 2019, de http://www.ditco.buap.mx/recursos/documentos/catalogopatentes2019.pdf

OCT-INDAUTOR (2019). Derechos de autor. Recuperado el 18 de mayo de 2019, de http://www.ditco.buap.mx/recursos/documentos/derechosautor_2019.pdf

OCT-PAD (2018). Benemérita Universidad Autónoma de Puebla. Resultados Convocatoria "Padrón de Consultores 2018". Recuperado el 14 de mayo de 2019, de http://www.ditco.buap.mx/recursos/documentos/resultadospadronconsultores2018.pdf

OCT-PAT (2019). Patentes solicitadas. Recuperado el 11 mayo de 2019, de http://www.ditco.buap.mx/recursos/documentos/patentes_2019_.pdf

OECD (2003), Turning Science into Business: Patenting and Licensing at Public Research Organisations, OECD Publishing, Paris, https://doi.org/10.1787/9789264100244-en.

OTT-CONACyT (2018). Oficinas de transferencia de tecnología (OTT) reconocidas por PROSOFT - Innovación, Fondo Sectorial de Innovación. Recuperado el 15 de abril de 2019, de https://www.conacyt.gob.mx/index.php/sni/convocatoriasconacyt/convocatorias-programa-de-estimulos-a-la-innovacion/pei-19/18209-directorioott/file

Pagano, U., \& Rossi, M. A. (2017). Economía del conocimiento, crisis financiera y depresión. Revista de Economía Institucional, 19(36), 57-74.

Pedraza Amador, Elba M. y Velázquez Castro, Judith A. (2013). Oficinas de Transferencia Tecnológica en las Universidades como Estrategia para Fomentar la Innovación y la Competitividad: Caso: Estado de Hidalgo, México. Journal of technology management \& innovation, 8(2), 221-234. 
Desempeño de una Ofícina de Transferencia de Tecnología en el contexto de gestión de patentes: Estudio de caso de la OTT de la Benemérita Universidad Autónoma de Puebla

PEI (2017). Programa de Estímulos a la Innovación. Recuperado el 2 de mayo de 2019, de https://www.conacyt.gob.mx/images/pdfs_conacyt/PEI_Beneficiarios_2009-2017.xlsx

Pérez Hernández, M. del P. M. (2016). Las organizaciones intermedias en los procesos de innovación en México. Perfiles latinoamericanos, 24(48), 161-183. http://dx.doi.org/10.18504/p12448-007-2016

Pérez, Hernández, P. y Calderón Martínez, G. (2014). Análisis de los procesos de comercialización de tecnología en dos instituciones de educación superior mexicanas. Journal of technology management \& innovation, 9(3), 196-209. http://dx.doi.org/10.4067/S0718-27242014000300015

Perkmann, M. y Walsh, K. (2007). University-industry relationships and open innovation: Towards a research agenda. International Journal of Management Reviews, 9(4), 259280. doi:10.1111/j.1468-2370.2007.00225.x

Rojas Arce, José L.(2017) Las Oficinas de Transferencia de tecnología y su papel en la estructuración de proyectos de innovación: El caso de una oficina mexicana. ALTEC 2017 XVII Congreso Latino-Iberoamericano de Gestión Tecnológica. Recuperado el 25/09/2019, de http://www.uam.mx/altec2017/pdfs/ALTEC_2017_paper_55.pdf

SE-CONACyT (2012). Guía para la certificación de una Oficina de Transferencia de Conocimiento (OT). Recuperado el 28 de abril de 2019, de http://20062012.conacyt.gob.mx/fondos/FondosSectoriales/FINNOVA/Documents/GUIA_DE_CER TIFICACION_OT.pdf

SE-CONACyT (2017). Convocatoria para el Reconocimiento de Oficinas de Transferencia de Tecnología (OTT). Recuperado el 15 de abril de 2019, de https://www.gob.mx/se/articulos/lanzamiento-de-la-convocatoria-para-el-reconocimientode-oficinas-de-transferencia-de-tecnologia-ott

SE-CONACyT (2018). Segunda Convocatoria para el Reconocimiento de Oficinas de Transferencia de Tecnología (OTT) Recuperado el 31 de julio de 2019, de https://www.conacyt.gob.mx/index.php/convocatorias-conacyt/convocatoriasconacyt/convocatorias-fondos-sectoriales-constituidos/convocatoria-se-conacyt-sectorialde-innovacion/convocatorias-abiertas-se-conacyt-sectorial-de-innovacion/17328-18-02seg-conv-ott 
Solleiro Rebolledo, J. L., \& Escalante Leyva, F. I. (2009). Knowledge management and value creation in Latin American research centers.

Villegas Cuadros, J. y Cárdenas Díaz, J. C. (2018). Spin-off como estrategia de transferencia de investigación a la industria: Estudio de caso Universidad EAFIT (Master's thesis, Universidad EAFIT).

WIPO. (2019). Wipo eLearning Center. Recuperado el 23/09/2019, de https://welc.wipo.int/

Yin, Robert. K. (1994). Case study research. Design and methods. California: SAGE Publications.

Yin, Robert. K. (2009). Case study research: design and methods (4 ed.). California: SAGE Publications. 